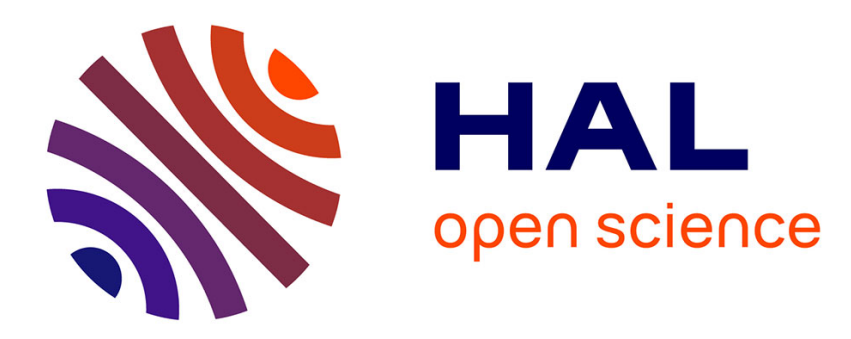

\title{
Fidélité et infidélité de la mémoire
}

Maurizio Balsamo

\section{To cite this version:}

Maurizio Balsamo. Fidélité et infidélité de la mémoire. Revue Française de Psychanalyse, 2013, (Vol. 77) (1). hal-01415309

\section{HAL Id: hal-01415309 \\ https://hal-univ-paris.archives-ouvertes.fr/hal-01415309}

Submitted on 12 Dec 2016

HAL is a multi-disciplinary open access archive for the deposit and dissemination of scientific research documents, whether they are published or not. The documents may come from teaching and research institutions in France or abroad, or from public or private research centers.
L'archive ouverte pluridisciplinaire HAL, est destinée au dépôt et à la diffusion de documents scientifiques de niveau recherche, publiés ou non, émanant des établissements d'enseignement et de recherche français ou étrangers, des laboratoires publics ou privés. 


\section{Maurizio Balsamo}

\section{Fidélité et infidélité de la mémoire}

Livio arrive à notre première séance en me communiquant immédiatement une sensation de surprise et d'incertitude. Il s'assied et reste longtemps là sans rien dire, en me souriant, mais d'un sourire inexpressif, indifférent, immobile. Je lui demande s'il peut me parler des raisons qui l'ont poussé à me demander un rendez-vous ; il évoque, avec peine, des « voix » qu'il entend, des statues sombres dans le couloir de son appartement qui essaient de se saisir de lui, des nuits troublées par des bruits infernaux, et le fait que chez lui, ça hurle sans arrêt. Puis il se tait, et il reste là à m'observer, en souriant tout le temps. Je reste là moi aussi, et je le regarde, je suis perplexe après avoir entendu ces maigres fragments, mais surtout à cause de cette rencontre qui ne me semble guère prometteuse, et dont les premiers échanges me semblent dénoter une lutte féroce entre le besoin de se confier et des vécus de possession, des sentiments angoissants d'aliénation et de paralysie. Il ajoute qu'il prend de l'héroïne, et puis il continue à me regarder fixement, toujours en souriant. Je réfléchis, j'écoute les quelques mots qu'il me dit, mais je me rends surtout compte que c'est un patient que je ne veux pas accepter, car je suis conscient - au moins à un premier niveau de réflexion - des efforts énormes qu'un travail avec lui exigerait de moi. Je continue à réfléchir, à part moi, à la façon de lui communiquer, éventuellement, mon refus, mais je reste quand même dans l'attente. Ce conflit entre accepter et repousser est si intense qu'il me détache de la situation et qu'il avive tellement mon intérêt, dans un mouvement autoérotique de narcicisation, qu'il me permet de recommencer à penser. Je me demande ce qui se présente sur la scène historique et relationnelle que nous sommes en train de vivre: je peux penser, bien entendu, à de violents mouvements d'expulsion de la rencontre que je vis comme par procuration, à des phénomènes d'identification projective, à des angoisses du contact que je prends en charge, pour des raisons personnelles et relationnelles, en laissant à l'arrière-plan, car c'est seulement les jours suivants que feront leur apparition - peut-être même comme trace d'une fonction antitraumatique - des questions sans réponse : en quoi ce que j'ai vécu à ce moment-là peut-il s'apparenter à une scène de véritable possession, ou de migration de processus archaïques? Et comme le conflit vers le rejet est vraiment intense, et que mes vécus m'intriguent, je décide de poursuivre nos séances.

Quelques mois plus tard, le patient révèle par petites touches, qui montrent en filigrane son interrogation sur le sens de cette histoire, que sa mère lui a fréquemment raconté - en lui proposant 
ainsi de manière répétée l'énigme et le traumatisme de cette fermeture sensorielle-perceptive - que jusqu'au sixième mois de grossesse, elle ne savait pas qu'elle était enceinte de lui et que personne ne s'était aperçu de quoi que ce soit dans sa famille. Il est indéniable que le patient essaie de penser le sens de ce récit : celui-ci pourrait aller de la suppression de son existence, à une théorie des origines férocement antisexuelle, au refus d'une intervention paternelle dans la naissance des enfants, ou encore à une invitation séductrice/aliénante à être comme il avait été dans le ventre : silencieux, immobile, fixé à un projet identificatoire qui lui aurait été assigné de manière précoce. $\mathrm{Ou}$ bien un récit qui était apparemment adressé au patient, mais qui avait en fait, comme interlocuteur secret, la mère de la mère du patient, à qui cette dernière s'adressait dans une réactivation de l'originaire maternel comme lieu indifférencié et indifférenciant.

Livio construit probablement, en même temps, une origine dont il n'est pas le destinataire mais l'organisateur occulte (quelqu'un qui se glisse secrètement dans le corps maternel, malgré celui-ci, contre celui-ci, plutôt que grâce à celui-ci). Bien entendu, on ne peut pas non plus sousestimer le collapsus dramatique des temps, où l'histoire de l'origine est effilée dans son impondérable complexité et où s'actualise inconsciemment, dans le présent de la séance - dans mon désir d'expulsion -, un aspect de celle-ci, qui détermine un comportement et un sentiment dans lequel l'analyste est appréhendé dans sa qualité métahistorique d'objet non contenant : au contraire, avortant, et avec lequel engager un corps à corps pour continuer à exister.

C'est dans cet ensemble complexe actualisé par le couple analytique, relatif à un «il ne faut rien dire sur la naissance », que s'inscrit peut-être aussi la tentative d'instituer un secret - celui de sa venue - qui permet de ne pas être expulsé/anéanti par l'autre, en créant un mythe psychotique des origines qui est le fruit d'une tromperie, plus que d'un désir partagé, grâce auquel Livio parvient à se glisser, à s'accrocher, dans une lutte désespérée pour la vie. En écoutant ce récit, je repense ainsi à mes vécus initiaux, à la lutte pour garder/expulser ce patient. En un certain sens, la fidélité de l'histoire des origines qui réapparaît en séance (avec de telles variations qu'elles réalisent la possibilité d'une différence dans le fonctionnement psychique par rapport à l'objet originel, et la construction d'un après-coup) apparaît sous la forme d'un vécu personnel, actuel, d'un certain point de vue dépossédant. Actuel aussi parce qu'elle n'a jamais appartenu à l'histoire du patient, si ce n'est sous la forme d'un élément scindé, impensé et qui attend de se réaliser dans une relation pour (pouvoir) devenir un élément de son passé. En même temps, cette fidélité est complètement infidèle, car elle est le fruit d'une sélection inconsciente des fils interprétatifs de cette même histoire, mais qui, de toute évidence, pour des raisons inconnues du couple, est sélectionnée comme le mythe des 
origines du couple, lequel doit donc se rencontrer autour d'une mémoire qui réorganise et qui réassemble l'histoire comme «un récit sacrificiel». Toutefois, ce «coup » est-il assimilable au récit de celui-ci qui arrive nécessairement après, en le liant autrement, en l'ouvrant à des destins interprétatifs? Ne pourrait-on pas dire alors qu'il y a déjà ici deux niveaux intéressants du fonctionnement psychique, où la fidélité d'un événement traumatique est accompagnée par l'infidélité nécessaire des significations et des tentatives de traduire l'absence en énigme ? L'effilochage interprétatif concernerait alors l'énigme, mais pas la force ni l'intensité d'un coup qui s'abat.

Une fidélité qui pourrait être pensée comme la trace (la mémoire ?) d'un événement traumatique qui se reflète dans l'attitude régressive de l'analyste qui écoute les mouvements de son ventre et qui appréhende, grâce à sa réalité émotive ainsi sollicitée, celle d'une histoire qui cherche une pensabilité et, bien entendu, la confirmation de son éternité intangible.

En même temps, cette fidélité se révèle être une illusion : elle n'assume en effet que l'un des fils de l'histoire de ce traumatisme, la «lutte pour/contre la vie », qui devient le mythe de fondation de notre relation, dans une sorte de collapsus qui efface et qui mélange tous les temps, tous les personnages et toutes les autres possibilités de lecture.

Il faudrait toutefois se demander ce qui permet, dans l'histoire du transfert, de réactualiser si puissamment cet originel (ou plutôt, une possibilité de celui-ci), de rendre présent le traumatisme, mais aussi de modifier le destin de cette histoire. C'est sans aucun doute l'analyste qui produit une réminiscence à la place du patient, en créant les conditions d'accès ultérieures, qui perçoit et qui accueille le «coup initial», l'avant-coup, en écoutant ses propres mouvements expulsifs, au moment où il vit cette «trace » et où il la réinscrit à travers l'écoute de ses propres mouvements intérieurs. C'est la circulation de cette énigme dans le récit d'un traumatisme offert un nombre incalculable de fois au patient, donc dans l'attente d'un destinataire possible, qui définit le champ de possibilité réalisé par l'analyse : c'est-à-dire que c'est l'analyste qui se trouve lui-même aux prises avec une énigme (celle de la violence de ses propres mouvements expulsifs), laquelle permet, de manière paradoxale, de traduire, en donnant cependant l'illusion de retrouver intacte une fidélité de l'origine. En même temps, toutefois, quel type de travail psychique doit-il se produire pour passer d'un grumeau de violence et de non pensabilité à une énigme, en rendant ce grumeau perceptible grâce à cette transformation ? En outre, quelle est la condition nécessaire pour transformer la perception de la condition de «stupeur » avec laquelle le patient se présente à moi, 
donc une histoire actuelle, qui pourrait être orientée de manière erronée uniquement par le (mauvais) fonctionnement d'un couple analytique, vers une mémoire possible quoique fallacieuse, (où l'actuel et le passé peuvent se confondre grâce aux difficultés de l'analyste, qui crée un tel incipit contre-transférentiellement) ? Comment passer d'un sourire sans sujet ni destinataire à la réplique d'une immobilité originelle (dotée de sens et d'émotion, une scène relationnelle et non plus solitaire) ? Ou alors il faudrait encore s'interroger sur la qualité du travail nécessaire pour que le temps puisse faire irruption dans une rencontre qui se prête, à travers le combat de l'analyste, à recevoir l'empreinte d'une infinité d'autres combats, d'une infinité d'autres non rencontres, en permettant d'arriver au récit de cette empreinte.

Ce qui implique que la fidélité est seulement un aspect de l'histoire, l'énigme résultant être la sexualisation de l'événement originel qui se réalise en séance, à partir d'une transformation de l'histoire en cours, d'un réinvestissement libidinisant qui permet d'actualiser un excès originel, de réinterpréter l'actualité d'un diagnostic comme une mémoire corporelle archaïque, en relisant l'avenir possible d'une expulsion comme un «déjà là »; une fixité psychotique, isolée dans la terreur, comme un accommodement tragique à un désir mortifère de la part d'un fœtus qui tait son existence et accepte $a b$ origine de disparaître pour pouvoir exister. Mais il faudrait aller plus loin, et se demander si le patient qui je garde/expulse dans mon esprit, est le même que celui qui est assis devant moi. Si dans la création/découverte de cet incipit, l'histoire de cette relation ne devait en quelque sorte que passer par là, en acceptant toutefois l'idée - comme dans Si par une nuit d'hiver un voyageur de Calvino - que d'infinis incipits sont possibles. Quel infans verra donc le jour ? Quel infans tue(-t-on) et lequel garde(-t-on) ? Quelles mémoires et combien de mémoires se sont-elles effondrées?

Comme on sait, percevoir et conserver, inscrire et mémoriser sont les thèmes du Bloc-notes magique, où à côté d'une surface qui conserve la trace de l'événement, s'affirme la nécessité de la présence d'une opération qui rende possible la réception du nouveau, en laissant à l'arrière-plan ce qui avait été enregistré et qui pourrait être relu aujourd'hui, d'un certain point de vue, à la lumière de l'hallucination négative comme la «structure encadrante » proposée par Green. Au contraire, c'est précisément dans les cas comme celui de Livio que l'originel fait irruption, que la mémoire s'impose sur le nouveau, en le pliant violemment à sa réapparition, que l'arrière-plan ne se constitue pas et que tout reste éternellement au premier plan. 
Mais la question du Bloc-notes magique, avec toute l'importance accordée aux processus d'inscription et, en même temps, avec cette méfiance initiale que manifeste le texte à l'égard de la mémoire ( Si je n'ai pas confiance dans ma mémoire - il est notoire que les névrosés s'en méfient beaucoup, mais les personnes normales aussi ont maintes raisons de s'en méfier », dit Freud), rend compte du fait qu'il existe - comme l'observait Le Guen ${ }^{1}$ - deux lignes, deux mouvements, deux approches nécessairement complémentaires à une théorie de la mémoire. D’une part, l'attention pour les fonctions de mise en mémoire, d'inscription et de conservation des traces, jusqu'à son extension neurophysiologique : la thèse selon laquelle tout ce qui est vécu est conservé, selon laquelle «dans la psyché rien ne se perd $»^{2}$, et qui prépare les étapes suivantes (pas nécessairement, mais aussi) d'un génétisme du développement qui instaure une forme de causalité évolutive/maturative. D'autre part, la thèse selon laquelle c'est l'étape finale qui décide des destins de la trace, dans un parcours de réinscription/transformation continuelle sur lequel seuls les déformations, les élaborations, les après-coups et les constructions sont les organisateurs finaux qui décident des destins possibles de l'antécédent ${ }^{3}$. D'une part, la trace d'un coup, avec sa valeur attractive-régressive ; de l'autre, les infinis après qui réécrivent sans fin ou qui échouent dans cette tentative, en signalant de la sorte une difficulté à digérer l'histoire et à l'englober selon le principe de plaisir, c'est-à-dire le principe d'assertion subjective. Mais il vaut la peine de préciser qu'en réalité, cette distinction est aujourd'hui trompeuse, parce que toute réactivation de la trace mnésique est à l'origine de sa fragilisation ; et donc que jouir de la conservation de la trace implique en même temps qu'on la condamne à la disparition.

Il n'en reste pas moins vrai que ces deux mouvements - celui de l'appui (de la trace) et celui de l'après-coup - se soutiennent l'un l'autre, et qu'à côté de la persistance des désirs inconscients comme l'observe Freud dans Inhibition, symptôme et angoisse -, il faut penser à la question de l'infidélité fondamentale de la mémoire, de sa retranscription continuelle: «..de la motion pulsionnelle refoulée elle-même nous admettions qu'elle subsistait non modifiée dans l'inconscient pendant une durée indéterminée. Maintenant l'intérêt se tourne vers les destins du refoulé, et nous pressentons qu'une telle persistance non modifiée et non modifiable ne va pas de soi» ${ }^{4}$. Ce type de réflexion indique que la thèse de l'inscription mnésique peut être pensée comme une résistance à accepter le fait que dans la dimension psychanalytique, c'est la lecture, la métabolisation - la

\footnotetext{
${ }^{1}$ «"Quand je me méfie de ma mémoire..." : essai pour en finir avec les théories de l'inscription », in Revue Française de Psychanalyse, vol. 45, $\mathrm{n}^{\circ}$ 5, 1981.

2 «Dans la vie psychique, rien ne peut périr une fois que cela s'est formé, tout d'une certaine manière se conserve », Malaise dans la civilisation (1929).

${ }^{3}$ Sur la question de la recatégorisation comme modèle du processus transformatif en analyse, cf. A. Modell, « Memory and the Psychoanalytic Cure », Canadian J.Psych., 2, 1994.

${ }^{4}$ Inhibition, symptôme et angoisse, Puf, Paris, p. 55.
} 
dynamique constructive, ajouterais-je - qui prévaut sur l'écrit (originel) et sur sa vérité historique présumée. Ou même, que les deux éléments fondamentaux de la mémoire peuvent être appréhendés dans le rapport existant entre la nécessité adaptative de la mémoire et le refoulement ${ }^{5}$, en déterminant une processualité complexe selon laquelle le retour du refoulé correspond non pas à un souvenir, mais à un symbole mnésique (déformé). Comme l'observe Green, nous pouvons alors penser au rapport entre l'adaptatif, la fidélité de la trace - à elle-même, pourrait-on dire -, et le refoulement, comme au rapport, en termes d'analogie, entre la mémoire physique ou biologique et la mémoire psychique, au sens que la mémoire psychique transforme un rapport d'analogie minimale en un rapport d'analogie maximal, avec conservation du rapport d'analogie, alors que dans la mémoire biologique, ce rapport se réduit à sa dimension extrême - phénomènes de duplication, de réplique, etc. Cette question se pose évidemment dans tous les cas où la mémoire psychique fonctionne sur le modèle de la mémoire biologique, autrement dit comme réplique, duplication, répétition, comme résistance au temps, comme fidélité indésirable. En quelque sorte, nous pouvons retrouver ici la distinction que Michel de M'Uzan avait instituée entre la répétition comme retour de l'identique ou du même ${ }^{6}$.

C'est au fond ce que Freud observe dans son texte sur les Souvenirs-écrans : «Chez bon nombre de personnes, les souvenirs d'enfance le plus anciennes ont pour contenu des impressions quotidiennes et indifférentes qui, lorsque 'elles ont été vécues, n'ont pas été en mesure de produire, sur l'enfant non plus, un effet à caractère affectif et qui pourtant ont été retenus dans tous les détails-on aimerait dire: avec une extrême netteté. ${ }^{7}$ La fidélité indésirable de la mémoire s'exprime au plus haut point dans les phénomènes de répétition : «Il est bien plutôt obligé de répéter le refoulé comme expérience vécue présente, au lieu de s'en souvenir comme d'un morceau du passé, ce que préférerait le médecin. Cette reproduction, apparaissant avec une fidélité qu'on $n$ 'aurait pas souhaitée.. ${ }^{8}$. La protestation contre cette fidélité révèle ainsi le parcours que le travail analytique doit accomplir en passant de l'identité de perception à l'identité de pensée, avec la renonciation à l'objet originel et à son aura. En même temps, Freud souligne différents aspects : l'événement traumatique, dans son insistance comme élément rebelle à la représentation, détermine, dans la tentative de retranscription qui est tout de même à l'œuvre dans le sujet humain, la construction de souvenirs- écrans, qui définissent de la sorte un à-côté, une liminalité qui permet de

\footnotetext{
5 «Quelques considérations sur les problèmes psychanalytiques de la mémoire », Évolution psychiatrique, 1974, vol. 39.

${ }^{6}$ Bien que l'identique ne soit jamais vraiment identifiable en tant que tel (nous devons accepter radicalement l'inconnaissabilité de la «fidélité originelle»), il reste toutefois important d'accepter son existence d'un point de vue métapsychologique.

${ }^{7}$ Freud, «Sur les souvenirs-écrans », in Huit études sur la mémoire et ses troubles, Gallimard, 2010, pag.87

${ }^{8}$ Au-delà du principe de plaisir, pag.289, Euvres complètes, Puf, vol.XV
} 
conserver/transformer la trace des réactions émotives, dans un effort de déplacement allusif, et qui produit, à travers la dimension mnésique, le perceptif nécessaire pour affronter la poussée régressive du traumatique à cause de sa non représentabilité.

L'observation du soi enfant apparaît d'ailleurs dans ces souvenirs, ajoute Freud, ce qui est évidemment impossible et «il est clair que cette image mnésique ne peut pas être la reproduction fidèle de l'impression reçue à l'époque.. une preuve que l'impression d'origine a subi une réélaboration ${ }^{9}$. L'événement mnésique qui est modifié, indique qu'une opération transcriptive s'est réalisée et qu'il faut passer par la modification du sujet qui en résulte pour retrouver le sens et la portée des processualités pulsionnelles/historiques. L'infidélité, la même qu'il revendique dans l'écriture de Mö̈se, son roman historique, permet de placer à l'arrière-plan la mémoire de ce que l'on connaît pour tisser différemment le fil de l'histoire, en mettant en évidence les transcriptions réalisées et le degré de fiction nécessaire pour pouvoir vivre et penser cette même histoire ${ }^{10}$.

Il est donc paradoxal que dans Constructions en analyse, Freud aille jusqu'à dire que nous désirons arriver dans le travail analytique «à une image fidèle des années oubliées par le patient », étant donné que pour appréhender cette fidélité, il faudrait passer à travers toutes les transcriptions, les déformations, les élaborations, les après-coups et les fragmentations de la matière psychique qui ont caractérisé l'histoire inconsciente du sujet. Certes, nous pourrions interpréter ce passage comme la persistance du projet freudien de résolution de l'amnésie infantile. Il faudrait évidemment se demander si le travail analytique «fidèle » proposé dans Constructions n'exprime pas en premier lieu la recherche non pas d'une fidélité des traces, mais des processus psychiques qui sont à l'œuvre dans l'enfance du sujet, et auxquels celui-ci aspire à retourner. Mais, surtout, si le travail analytique ne montre pas la tentative d'actualiser une autre fidélité, c'est-à-dire qu'il est soumis à une dynamique régressive et antiélaborative, celle qui impose à la trace de ne pas réapparaître sous peine de catastrophe (cf. la question posée par Winnicott sur la peur de l'effondrement), et qui reste fidèle à elle-même pour éviter d'être prise en charge par les processus d'historicisation, en circulant dans les processus associatifs qui usent la trace ${ }^{11}$. Autrement dit, la fidélité est le signe non pas d'une vérité de l'expérience, mais d'une série complexe d'opérations psychiques, inhérentes à des dynamiques constructives/retranscriptives, qui instituent, par exemple, une dimension traumatique originelle comme élément perceptif auquel raccrocher sa propre histoire identitaire, et qui fondent

\footnotetext{
${ }^{9}$ Souvenirs- écrans, cit, pag. 112.

${ }^{10}$ Apparaît en même temps une relation entre la viscosité de la libido et la mémoire trop fidèle, comme écrive Freud in Analyse terminée et analyse interminable.

${ }^{11} \mathrm{Cfr}$. les rapports entre usure et association dans les Études sur l'hystérie.
} 
un parcours objectualisant, en permettant de s'ancrer au connu, en évitant de la sorte d'affronter ce que l'on ne connaît pas, cette terreur de l'inconnu qui devrait frapper les deux partenaires d'une analyse, comme le disait Bion, sous peine de l'inutilité de celle-ci ${ }^{12}$.

\section{Usure/non usure}

Dans les réflexions sur l'hystérie (cf. Le mécanisme psychique des phénomènes hystériques), où Freud parle des représentations devenues pathogènes qui gardent leur dimension affective parce que est nié l'usure à travers l'abréaction, ou bien, par exemple, dans L'Homme aux rats, Freud réfléchit sur l'usure, la perte et la consommation que le souvenir reçoit dans le passage à la conscience et au temps. Toutefois, si le système associatif, la dynamique représentationnelle permet l'usure et l'affaiblissement de la force pulsionnelle, il existe des phénomènes et des traces dont le destin est d'être détaché de cette possibilité «parce que on ne peut pas se souvenir de ces traces ${ }^{13}$, en montrant un double destin/registre des traces mnésiques : celles qui peuvent se « consommer » et celles qui se soustraient radicalement à ''usure $^{14}$, parce qu'elles opèrent dans une fonctionnement psychique d'où nous pourrions dire que le sujet est absent, où il n'y a rien à se rappeler et donc rien à consommer. La fixité de la trace, sa non dégradation renverrait donc à deux destins différents : l'inaltérabilité (relative) de l'inconscient et son usure, et la soustraction radicale au temps des traces dont on ne peut pas se souvenir, et qui s'imposent donc dans un statut différent de celui de l'inconscient refoulé. Il semblerait alors qu'au moins ici, la fidélité ne soit pas le fruit d'une constructivité en tant que facteur inhérent à la recherche de moyens pour consolider le fonctionnement hallucinatoire en l'absence de poussée désirante.

Toutefois, c'est seulement en apparence que cette question se pose en des termes si nettement dichotomiques : le devoir de mémoire, par exemple, est une nécessité pour que l'on

\footnotetext{
${ }^{12}$ En outre, cette fidélité du manque à laquelle la construction devrait ensuite répondre, comme dimension palliative ou prothésique, substitutive, peut-elle être vraiment pensée comme exempte de processus de désexualisation qui déterminent des objets manquants plutôt que conflictuels, des blessures originaires plutôt qu'oedipiennes, des conditions d'impuissance infantile, au lieu de confrontations avec la dimension complexe de l'adulte et de sa vie affective et sexuelle ? Il s'agit ici d'une extension abusive du modèle freudien ou d'une lutte contre la sexualité, dans la tentative de conserver le secret des fixations sexuelles et génitales, telle qu'elle a également été posée par Green à propos des constellations limites et du fait qu'elles sont toujours interprétées sur le versant d'un archaïque désexualisé ? A. Green, «La sexualité a-t-elle un quelconque rapport avec la psychanalyse ?», Revue franc. de psych., 1996, 3. Et cela sans négliger le fait que la fidélité de ces conditions réside dans la fixation inexorable à l'objet primaire, en rendant vain tout espoir d'un déplacement des investissements, d'un destin différent de sa propre histoire engloutie par une mémoire du désespoir.

${ }^{13}$ Études sur l'hystérie

${ }^{14}$ On trouvera une réflexion plus vaste sur l'usure des traces dans Mécanisme des phénomènes hystériques (1893).
} 
n'oublie pas et que l'Histoire ne se répète pas; mais ce peut être aussi un instrument pour consolider une identité paranoïaque organisée sur le couple ami/ennemi ${ }^{15}$. L'excès de mémoire n'est pas seulement un excès reçu, au sens d'une transmission ou d'une contrainte aliénante d'identité et de rôles, mais c'est aussi la tentative du psychisme de faire face à des poussées régressives grâce à la découverte/construction continuelle de souvenirs, de façon à créer des ancrages perceptifs qui fonctionnent comme autant de points d'arrêt et de lien identitaire. De manière analogue, l'excès peut être compris comme la compréhension d'une contrainte continue du psychisme, obligé de produire des réminiscences, sous forme de remémoration, de répétition, de constructions, dans le but de réaliser une inhibition relative des activités régressives, en premier lieu le rêve, en permettant ainsi le travail de figurabilité psychique. «Ils fournissent à ces activités psychiques des matériaux pour que le travail qui le caractérise, le travail de rêve, le travail de symptôme, le travail d'incidence, celui fondant les pensées incidents de séance, puisse se réaliser $\gg^{16}$.

En outre, qu'en est-il du statut de la mémoire dans les versions de l'analyse centrées essentiellement sur l'ici et le maintenant, avec le risque de perdre la polysémie des représentations inconscientes ${ }^{17}$, la pluralité des objets transférentiels, dans une lecture et un fonctionnement qui renvoient davantage au préconscient qu'à l'inconscient ? Ou avec le risque, tout aussi considérable, d'un couple qui se fossilise dans l'analyse de son propre fonctionnement, dans une processualité, ou dans une collusion infinie, uniquement attentif aux problèmes de survie de la relation et ignorant le fait que la recherche continuelle d'un accord émotif puisse n'être qu'une défense de la dimension régressive et de l'opération de déni qui caractérise toute processualité constructive (le manque que la construction doit pallier) ? Enfin, ne devrions-nous pas supposer un troisième registre de fonctionnement, qui rend partiel le système usure/non usure, autrement dit une «mémoire sans souvenirs », une modalité qui semble être la prérogative d'une clinique où la névrose de transfert, la temporalité classique et les différences de topiques ne semblent plus être en évidence et où la remémoration ne se produit en raison de l'entrée en scène de la question de la «conviction » à la place du souvenir, la problématique régressive et hallucinatoire mise en place par Constructions en analyse ? Dans ce texte - et S. et C. Botella y ont insisté tout particulièrement -, Freud propose une nouvelle conception de la mémoire «avec un retour hallucinatoire des éléments du passé qui ne sont

\footnotetext{
${ }^{15}$ Cf. « Devoir de mémoire : entre passion et oubli », Revue française de Psych., 2000, 1, 64.

${ }^{16}$ B. Chervet, «La théorie de la réminiscence généralisée et l'infantile », in Avancées de la psychanalyse, Paris, Puf, 2008, p. 144.

${ }^{17}$ Cf. A. Costa, « Memoria e relazione analitica », Riv. di psicoanalisi, 1981, 27.
} 
pas remémorables $»^{18}$. En partant de la question soulevée par la régressivité du fonctionnement analytique en séance, il s'agit d'accéder à un passé anhistorique, un «passé qui n'a pas encore été éprouvé » et pour lequel les notions d'actualisation (de « résurrection », devrions-nous dire) ${ }^{19}$, de figurabilité, d'hallucinatoire, d'une transformation d'un matériau qui n'est plus appréhendé dans sa qualité de souvenir, d'acte ou de sensation ou qualifiable temporellement, mais qui est globalement perçu dans son indifférenciation, comme matière psychique à revivifier, à re-rêver et transformer, sont de plus en plus importantes ${ }^{20}$.

La question de la double fidélité, celle qui est exprimée par les Constructions en analyse, et la fidélité originelle d'une mémoire qui ne semble pas décidée à se consumer, pose la question suivante : de quel type de trace s'agit-il dans cette fidélité absolue, soustraite (radicalement ?) à l'usure ? Cette fidélité à laquelle le travail analytique doit tendre, apparaît pensable comme la répétition en cours, en séance, d'un échec originel qui insiste pour réapparaître dans son identité de perception. On pourrait se demander si cette fidélité avec laquelle coïncider ne peut pas être appréhendée comme la présentation non pas des traces mnésiques (qui font partie des chaînes associatives avec la possibilité d'être modifiées), mais de celles qui prennent chez Freud le statut de «traces mémorielles », ou de traces perceptives ${ }^{21}$, c'est-à-dire de traces qui ne sont pas liées au Moi et qui constituent le fond psychique des souvenirs. «Une trace mémorielle peut acquérir la qualité image mnésique du souvenir représenté si elle est 'liée' par le Moi et, sous cette forme, peut entrer à faire partie des réseaux des représentations. ${ }^{22}$ On voit s'ouvrir ici, en particulier, un immense domaine de recherche concernant le statut de la «conviction à la place du souvenir » et du sentiment de réalité effective comme fondement de la construction en après-coup d'ébauches mnésiques pour un appareil psychique en l'absence de celles-ci. Comme je n'ai pas le temps ici d'examiner cette question, je ne peux que renvoyer à un certain nombre de travaux sur ce sujet ${ }^{23}$. Toutefois, si ces traces sont le résultat d'une perception, de l'affect (de la sensation) et du type

\footnotetext{
${ }^{18}$ C. Botella, «Les niveaux de mémoire et de vérité : leur interprétation », Bolletino della Fep, 2005, 59 ; C. Botella, «La mémoire sans souvenirs », in Avancées métapsychologiques, l'enfant, la famille, 1991; S. Botella, «Pour la métapsychologie de la remémoration », in Les voies nouvelles de la thérapeutique, Paris, Puf, 2006 ; M. Mancia (2006). «Memoria implicita e inconscio précoce non rimosso : loro ruolo nel transfert e nel sogno », Rivista di Psic., 52 ; T. Bastianini, G. Moccia (2003), «Riflessioni sulle attuali evoluzioni dei concetti di affetto, memoria e azione terapeutica », Riv. di Psicoanal., 2003, XLIX, 3 ; G. Moccia, «Le memorie identificatorie nell'organizzazione del transfert », in (sous la direction de A.M. Nicolò), Attualità del transfert, Milan, Franco Angeli, 2009.

${ }^{19} \mathrm{~S}$. Botella, «La naissance de la pulsion. Les processus originaires et la pratique analytique », Revue française de Psych., 1, 2007.

${ }^{20}$ Cf. aussi D. Quinodoz, «Comment construire quand la remémoration est impossible ? », Revue française de Psych., 2008, 5.

${ }^{21}$ R. Roussillon, « Historicité et mémoire subjective. La troisième trace », Cliniques méditerranées, 67, 2003.

${ }^{22}$ S. Botella, « Pour une métapsychologie de la remémoration », cit. p. 713.

${ }^{23}$ C. et S. Botella, «Pensée animique, conviction et mémoire », Revue française de Psych., 4, 1985 ; B. Chervet, « Constructions, détresse et conviction en psychanalyse », Revue française de Psych., 2008, vol. 72.
} 
d'investissement ou de désinvestissement (avaler/cracher) qu'elles enregistrent, et si elles renvoient à des expériences répétées, elles cessent alors d'être des enregistrements, qui seraient d'ailleurs difficilement présents dans la vie psychique, pour devenir des traces catégorielles, des schémas de relation, à très bas quotient de transformation, qui organisent l'histoire du sujet. Il vaut la peine d'observer que dans le cas où le travail du Moi fait défaut, c'est alors la dimension délirante qui prend en charge les processus de lien/mémoire.

\section{Histoire/mémoire}

Si nous pouvons imaginer que la Mémoire peut avaler l'Histoire, en la gélifiant, nous devons aussi penser - comme l'observe correctement Garella ${ }^{24}$ - que dans le cas des représentations d'interactions généralisées, forme de mémoire procédurale organisationnelle, prédictive et rétrodictive, il est vrai que non seulement l'Histoire produit de l'inertie dans le cas de la permanence en cours de ces mémoires conservatrices, mais que l'inertie, malgré elle, ne peut pas ne pas produire de l'Histoire. Ce qui amène à penser que dans la circularité entre l'organisation et ses formes d'actualisation, il se définit nécessairement un circuit rétroactif (quoique, comme je le disais, à bas quotient de transformation) capable de produire quand même des variations, des flexions et des écarts par rapport à la trace originaire. Il est également vrai, en outre, que si les traces deviennent des coalescences de relations et de modalités de réponses, il n'y a alors aucune fidélité de l'expérience de chacun, car ces traces définissent plutôt des classes ou des ensembles d'interactions qui définissent à leur tour les conditions de possibilité de l'événement et des moyens de le vivre.

Comme je l'ai observé précédemment, l'échec des processus transcriptifs apparaît de manière évidence dans toutes les fonctions «commémoratives » de la mémoire, dans leur recours à la fidélité de l'événement et à sa place chronologique, à l'unicité et à l'extranéité subjective de l'événement et à la construction d'un temps articulé autour d'une théorie traumatique. Qu'on pense, comme observe Freud, à cette femme dont il parle dans les Études sur l'hystérie, qui effectue des périodiques commémorations chaque année à l'époque des catastrophes, et dont la reproduction visuelle et affective est fidèlement liée à la date. On trouve une question analogue dans les Cinq essais sur la psychanalyse, où Freud compare le comportement de la réminiscence hystérique et

\footnotetext{
${ }^{24}$ A. Garella, «Coazione a ripetere e memoria », Riv. di psicoanalisi, 37, 1991. Cf. aussi le bel «Oublier Freud ? » de D. Scarfone, Montréal, Boréal, 1999.
} 
l'attitude mélancolique qui s'instaure devant un monument historique, symbole commémoratif de la ville, comme si un citadin, dit-il, continuait à pleure des événements qui se sont produits il y a très longtemps et auxquels se réfèrent les monuments. Dans ce cas, la mémoire «s'effondre » sur l'histoire et il me semble vraiment que le problème de la disjonction/rapport entre l'histoire et la mémoire - consistant à comprendre que leur identification mène nécessairement à la construction unique de l'histoire, à sa capture par une interprétation univoque, à l'effacement des possibles inhérents à celle-ci, à la prise en charge d'une construction qui se fortifie dans les souvenirs et dans les célébrations de ses anniversaires -, est une des questions les plus importantes du travail analytique. Et il convient sans doute d'observer que Freud multiplie les usages de l'Histoire, qu'il la fragmente en une multiplicité d'Histoires (du refoulement, de la guérison, de la cure, de la maladie, des la névrose infantile, etc., comme pour indiquer l'impossibilité d'une cohérence réductrice, les strates multiples où le sujet vit ou qui le possèdent). Nous pourrions donc dire que la Mémoire peut être un support de l'Histoire, dans sa qualité vivante, de mémoire signifiante, réticulaire, associative, élaborative, ou, au contraire, qu'elle peut avaler l'Histoire (parfois tendanciellement, parfois de fait, comme par exemple dans toutes les conditions qui prennent la valeur d'événements destinaux), la rendre stérile, en faire la pure gardienne du déjà écrit ou du déjà survenu ${ }^{25}$.

Il apparaît donc compréhensible que chez certains patients, la temporalité soit une dimension qui doit être entièrement construite et que la cure pourrait être lue comme la tentative - dans sa capacité de reviviscence, ainsi que de réminiscence - d'actualiser les traces perceptives, en les transformant en souvenirs, narrations, fantasmes, mythes, et en les condamnant enfin à l'oubli transformatif. C'est dans ces termes que le Passé peut devenir Histoire, et l'Actuel, Passé, grâce à la construction, dans le «nécessaire» de la processualité automatique et pré-subjectale, du « possible » du sexuel infantile et de sa temporalité anhistorique. Si l'on pense qu'Aristote situait en revanche le nécessaire dans le Passé et le possible dans le Futur, on peut, je crois, observer la portée révolutionnaire de la perspective freudienne, sans pour autant rabaisser la valeur de ce qui a trait à la création du nouveau dans la relation analytique. Nous pouvons espérer ainsi avoir accès à une autre mémoire, celle qui permet enfin de passer de l'actuel de la répétition à la réalisation d'une temporalité humanisante ; de ce qui est inscrit dans le passé de chacun de nous et qui attendait en souffrance, comme promesse manquée, désir irréalisé, choix non accompli, énigme, futur possible, rêve. Ou, encore plus radicalement, comme mémoire de ce que nous n'avons jamais pu (encore) vivre : autrement dit, construire le Passé.

\footnotetext{
${ }^{25}$ Une position analogue, reposant cependant cette fois sur le couple mémoire/désir, avait été exprimée par Corrao : "Mémoire et désir peuvent être considérés comme le "sens" passé et le "sens" futur d'une même chose », F. Corrao, «Memoria e oblio », Riv. di psicoanalisi, 1982, 28.
} 